\title{
O EFEITO DA ATIVIDADE FÍSICA NO APARELHO LOCOMOTOR DO IDOSO
}

\author{
THE EFFECT OF PHYSICAL TRAINING ON LOCOMOTIVE \\ APPARATUS IN ELDERLY PEOPLE
}

André Pedrinelli', Luiz Eugênio Garcez-Leme ${ }^{2}$, Ricardo do Serro Azul Nobre ${ }^{3}$

\section{RESUMO}

Alterações fisiológicas que ocorrem no aparellho locomotor devido ao envelhecimento como perda de massa muscular, perda do equilíbrio corporal, diminuição da massa óssea e osteoartrose causam limitações às atividade da vida diária do idoso, comprometendo sua qualidade de vida e o tornando mais frágil e dependente. Idosos que realizam atividade física periodicamente tem melhor independência funcional e melhor qualidade de vida do que aquele sedentário. Esse artigo aborda as principais alterações fisiológica do processo de envelhecimento e realiza uma revisão da literatura atual sobre os efeitos que o exercício físico causa no aparelho locomotor do idoso, especificando qual a melhor forma de prescrever atividade física nessa faixa etária.

Descritores - Envelhecimento; Saúde do idoso; Exercício; Atividade motora

\section{ABSTRACT}

Physiological changes taking place on the locomotive apparatus as a result of aging, such as muscular mass loss, body balance loss, reduced bone mass and osteoarthrosis cause limitations to the daily activities of elderly people, compromising their quality of life and making them weaker and dependent. Aged people who regularly practice physical activities have a higher level of functional independence and a better quality of life than the sedentary ones. This article addresses the key physiological changes with aging and provides a review of current literature about the effects of physical exercises on the locomotive apparatus of elderly individuals, specifying the best ways to prescribe physical exercises to this age group.

Keywords - Aging; Health of the elderly; Exercise; Motor activity

\section{INTRODUÇÃO}

O processo de envelhecimento biológico determina alterações no aparelho locomotor, que causam limitações às atividades da vida diária e, assim, comprometem a qualidade de vida da pessoa que envelhece. A diminuição do nível de atividade pode levar o idoso a um estado de fragilidade e de dependência. Evidências atuais demonstram que a atividade física traz benefícios à saúde do idoso, mantendo independência funcional ${ }^{(1)}$ e melhorando sua qualidade de $\operatorname{vida}^{(2)}$.

Projeções populacionais ${ }^{(3)}$ estimam que a população idosa brasileira ampliará sua importância relativa de
5,4\% em 2000 para 18,4\% em 2050 e que a pirâmide etária evoluirá em processo de estreitamento de sua base, caracterizando envelhecimento da população.

Dentre as queixas relacionadas com o sistema musculoesquelético, a osteoartrose (OA) é a doença articular mais prevalente entre os indivíduos acima dos 65 anos $^{(4)}$. Aproximadamente metade das pessoas com mais de 65 anos apresenta OA; sua prevalência aumenta para 85\% nos com mais de 80 anos $^{(5,6)}$. Estudos referem que $27 \%$ da população entre 63 e 70 anos apresentam sinais radiológicos de OA do joelho ${ }^{(7)}$. A OA é uma das principais causas de doença crônica e de incapacidade na população idosa ${ }^{(8)}$.

1 - Professor Colaborador da Faculdade de Medicina e Médico Assistente do Grupo de Medicina do Esporte do Instituto de Ortopedia e Traumatologia do HC-FMUSP.

2 - Médico Geriatra; Professor Associado do Departamento de Ortopedia e Traumatologia da Faculdade de Medicina da USP.

3 - Médico Colaborador do Grupo de Medicina do Esporte do Instituto de Ortopedia e Traumatologia do HC-FMUSP. 
A perda da massa muscular é outro aspecto importante a ser abordado. A perda da força e da potência musculares leva à diminuição na capacidade de promover torque articular rápido e necessário às atividades que requerem força moderada, como: elevar-se da cadeira, subir escadas e manter o equilíbrio ao evitar obstáculos. Isso, além de causar maior dependência do indivíduo, pode facilitar as quedas. Aproximadamente $30 \%$ das pessoas com mais de 65 anos e metade das com mais de 80 anos sofrem uma queda a cada ano ${ }^{(9)}$. A queda ou a lesão decorrente dela pode ter efeito devastador na independência do indivíduo e em sua qualidade de vida. Dentre as consequências das quedas podemos citar: lesões musculoesqueléticas (sendo a mais grave a fratura do fêmur proximal), o posterior medo de nova queda, a diminuição geral das atividades da vida diária, o deterioramento funcional, o isolamento social, a diminuição da qualidade de vida, a institucionalização e mesmo o óbito ${ }^{(10,11)}$. A queda associada à fragilidade óssea caracterizada pela osteopenia/osteoporose pode aumentar a probabilidade de o idoso sofrer algum tipo de fratura. Apesar de a fratura do quadril ocorrer em somente 1\% das quedas, ela é responsável por grande parte da incapacidade, das mortes e dos custos médicos relacionados aos tratamentos no idoso ${ }^{(12)}$.

O objetivo deste artigo é explicar como a atividade física pode trazer benefícios ao aparelho musculoesquelético dos idosos e expor quais são os exercícios usualmente indicados para essa população, numa revisão da literatura atual.

\section{EFEITOS FISIOLÓGICOS DO ENVELHECIMENTO}

O processo de envelhecimento está associado à perda de massa muscular (sarcopenia) e à correspondente redução da força muscular máxima ${ }^{(13)}$. A perda de massa muscular inicia-se aos 30 anos, mas aumenta ao redor dos 50, ocorrendo mesmo no indivíduo atleta ${ }^{(14-16)}$. Ocorre um fenômeno no qual a proteína contrátil é substituída por lipídios intra e extracelulares e por proteína estrutu$\mathrm{ral}^{(17)}$. Entre os 65 e 84 anos, a força isométrica muscular diminui aproximadamente $1,5 \%$ por ano, enquanto a potência muscular decresce $3,5 \%$ por ano ${ }^{(18)}$. A perda da potência muscular é maior que a da força devido à perda seletiva das fibras tipo II (fibras de contração rápida). A diminuição da potência muscular determina diminuição da velocidade de contração muscular e esta perda nos músculos responsáveis pela manutenção da postura ortostática pode contribuir para o aumento do número de quedas $^{(19)}$. Neurologicamente, existe diminuição, eventualmente modulada por sinais aferentes das articulações comprometidas (inibição artrogênica), do número e do tamanho das unidades motoras e isso é também uma causa primária da perda da força muscular ${ }^{(20)}$.

A perda da capacidade de reter água pelo organismo, devido ao envelhecimento, associada à diminuição da capacidade para produzir proteoglicanos, causa alterações degenerativas articulares, já que a cartilagem tem menor capacidade de absorver o impacto. Isso, associado à diminuição da estabilidade articular, da fraqueza muscular crescente e do aumento de peso, fisiológico com a idade, é fator de piora das alterações degenerativas $^{(21)}$. A propriocepção também declina com a idade e alguns estudos demonstram que essa perda também contribui para o desenvolvimento da $\mathrm{OA}^{(22,23)}$, porém, se questiona se a redução da propriocepção é causa ou consequência da $\mathrm{OA}^{(8)}$.

Ainda há alterações na resistência do sistema musculoesquelético caracterizadas por modificações estruturais dos tendões, que se tornam mais rígidos e, consequentemente, com maior possibilidade de sofrer microrrupturas ou mesmo rupturas completas ${ }^{(24)}$, e diminuição da massa óssea, causadas por desproporção entre as atividades dos osteoclastos em relação aos osteoblastos, havendo maior consumo e/ou menor produção óssea. Na mulher, nos primeiros anos pós-menopausa, o desequilíbrio nessa relação é mediado pela insuficiência de estrógeno. Mais tardiamente, à medida que aumentam as necessidades de cálcio com a idade, as deficiências desse e de vitamina $\mathrm{D}$ adquirem maior importância do ponto de vista etiológico. No homem, é causado pela diminuição dos níveis de testosterona, que ocorre de forma mais lenta e gradual. Dependendo da quantidade da perda óssea, o idoso pode apresentar osteoporose, que é uma doença caracterizada pela baixa massa óssea e deterioração da microarquitetura do tecido ósseo, levando a estado de fragilidade do osso e, consequentemente, a aumento do risco de ocorrência de fraturas ${ }^{(25)}$.

\section{BENEFÍCIOS DA ATIVIDADE FÍSICA PARA A POPULAÇÃO IDOSA}

Ao pesquisar a literatura sobre o impacto da atividade física regular no aparelho locomotor da população idosa, encontramos, principalmente, estudos que avaliam o efeito do exercício físico no equilíbrio corporal do individuo idoso e na população que apresenta OA. Com relação à osteoartrose, os estudos concluem que o treinamento 
físico não tem impacto sobre o processo fisiopatológico da doença ${ }^{(26-29)}$ e que ele é efetivo no controle da dor e na melhora da função do idoso ${ }^{(30)}$. Já para o equilíbrio, os estudos relacionam a atividade física com a melhora na estabilidade postural e na marcha do idoso, o que pode reduzir as quedas nessa faixa da população ${ }^{(31,32)}$.

Revisões sistemáticas que avaliam os efeitos do exercício físico na população com OA concluem que há benefício na dor e na função do idoso, porém, variam quanto à magnitude do efeito e não esclarecem quais tipos de exercícios teriam efeito maior. A revisão sistemática de Fransen e McConnell ${ }^{(33)}$ concluiu que a prática de atividades físicas no solo tem efeito pequeno, porém significante, na melhora da dor e na função do idoso com OA do joelho e é comparável aos benefícios causados pelo uso de analgésicos comuns e anti-inflamatórios não hormonais. Ainda nesse estudo, não há diferenças significantes entre os exercícios aeróbios comparados com o fortalecimento muscular na melhora da dor e da função; ainda, os exercícios supervisionados (tanto individual, como aulas em grupos) têm efeito maior no controle da dor do que aqueles não supervisionados. Por fim, conclui que recomendações específicas quanto ao programa de treinamento (intensidade, volume, frequência, progressão e duração) não podem ser feitas, por não haver nenhum estudo que avalie essas variáveis especificamente. Roddy et $\mathrm{al}^{(34)}$ fizeram uma revisão sistemática comparando caminhada aeróbia versus fortalecimento muscular localizado e concluíram que ambos reduziram a dor e a incapacidade causada pela OA, sem diferença estatística entre ambos; destacam no final que há grande variabilidade na duração dos programas de treinamento nos diversos estudos avaliados (de oito semanas a dois anos). O estudo de Woodard e Berry ${ }^{(35)}$ afirma que é necessária a aderência por longo tempo ao programa de exercícios para que se mantenha o benefício que a atividade física proporciona e que o estímulo de supervisão ou monitoramento regular pode ser uma alternativa para os idosos manterem a aderência ao tratamento. A meta-análise de Hernández-Molina et al ${ }^{(36)} \mathrm{e}$ a revisão de Tak et $\mathrm{al}^{\left({ }^{(37)}\right.}$ avaliaram o efeito do exercício para a OA do quadril e concluíram que a atividade física é eficaz no tratamento da dor, ressaltando a necessidade da presença do fortalecimento muscular no programa. Por fim, a revisão de Bartels et $\mathrm{al}^{(38)}$ avaliou o benefício dos exercícios aquáticos no tratamento da OA do joelho e quadril e concluiu que esse tipo de exercício apresenta efeitos benéficos de curto tempo para dor e para função; efeitos de longo tempo não foram documentados nos estudos avaliados. Dessa forma, os exercícios na água devem ser utilizados como parte inicial de um programa longo de exercícios para o paciente com AO.

Vários estudos mostram que o treinamento físico tem efeitos significativos sobre a massa muscular e sobre a força desenvolvida pelos músculos ${ }^{(39-43)}$. No indivíduo idoso está claro que a potência e não a força muscular é o fator determinante para a melhora da independência e da qualidade de vida ${ }^{(44,45)}$, além de ser melhor indicador para a prevenção do risco de quedas ${ }^{(46)}$. Assim, no idoso, é necessário que o fortalecimento muscular seja feito em alta intensidade. Alguns estudos com fortalecimento de baixa intensidade resultaram em aumento da força muscular ${ }^{(11,47)}$, porém, com ausência ou efeito muito baixo na saúde do idoso ${ }^{(48,49)}$. Apesar de o fortalecimento muscular levar a melhora da função, outros exercícios mais funcionais são necessários para haver ganho maior no equilíbrio e na independência do idoso ${ }^{(11,12)}$. Orr et $\mathrm{al}^{(50)}$ realizaram uma revisão sistemática que avaliou a eficácia do fortalecimento muscular progressivo sobre o equilíbrio corporal e sugerem que esse tipo de treinamento realizado isoladamente não é efetivo para a melhora do equilíbrio, acrescentando que outros fatores estão envolvidos na estabilidade postural. Skelton e Beyer ${ }^{(12)}$, ao realizar uma revisão da literatura, concluíram que o programa de treinamento para reduzir o risco de quedas e de fraturas após estas deve incluir exercícios de equilíbrio, de coordenação e de tempo de reação, além do fortalecimento muscular; e que treinamentos contendo só musculação ou caminhadas são menos efetivos para a estabilidade postural. Porém, Howe et $\mathrm{l}^{(51)}$, numa revisão sistemática, afirmam que diversos tipos de exercícios trazem benefícios ao equilíbrio corporal, mesmo se realizados isoladamente. Entre essas atividades físicas incluem: exercícios funcionais (para balanço, marcha e co-coordenação), fortalecimento muscular, caminhada, dança, tai chi chuan, bicicleta (tanto estacionária quanto livre); essa última apresentou apenas tendência de melhora no equilíbrio corporal. Porém, advertem que todas essas intervenções têm efeitos somente a curto prazo e que estudos padronizados e a longo prazo devem ser feitos para avaliar o efeito da atividade física regular na estabilidade postural.

Algumas formas de exercícios têm-se mostrado benéficas para retardar ou reverter a perda de massa óssea relacionada com a idade ${ }^{(52)}$. Entre essas atividades incluem-se: exercícios com peso ${ }^{(53)}$, rrrida $^{(54)}$ e aulas em academias ${ }^{(55)}$. Caminhadas isoladas não aumentam 
a densidade óssea, porém, ajudam a mantê-la ${ }^{(52)}$. Nakatsuka et $\mathrm{al}^{(56)}$ mostraram em seu estudo que a atividade física de moderada intensidade pode aumentar a massa óssea pós-menopausa, porém, o ganho era modesto e específico para determinadas regiões do corpo. Numa revisão sistemática de Bonaiuti et $\mathrm{al}^{(57)}$ avaliou-se o efeito de diversos tipos de atividade física na prevenção e no tratamento da osteoporose na mulher com menopausa. Os resultados mostraram que há evidência de que o exercício físico é efetivo para a diminuição da perda de massa óssea na coluna lombar e, provavelmente, no colo do fêmur e no punho. Tanto os exercícios aeróbicos, como os de fortalecimento muscular e a caminhada tiveram diferença significante, porém, ressalvam que não há uma conclusão de qual é a característica do exercício (tipo, intensidade, frequência ou duração) que traz maior benefício para a massa óssea e nem se esse benefício persiste após a suspensão das atividades físicas. Korpelainen et $\mathrm{al}^{(58)}$, num estudo populacional randomizado e controlado, concluíram que a realização de exercícios físicos teve um efeito positivo especialmente na massa óssea do trocanter e que podem evitar as fraturas relacionadas com a queda na mulher idosa. Karinkanta et al ${ }^{(59)}$ verificaram que os benefícios induzidos pelo exercício no equilíbrio dinâmico e na massa óssea da tíbia persistem após um ano do término da atividade física, apesar de não se manterem na força muscular e na função física.

\section{PRESCRIÇÃO}

Como já discutido anteriormente, a combinação ótima de exercícios para haver melhora da função do aparelho locomotor no idoso é ainda desconhecida. Atualmente, existe somente um pequeno suporte empírico, que combina o fortalecimento muscular com outros modos de treinamento (aeróbio, exercícios de equilíbrio e de coordenação). A intensidade, a duração, a frequência e a progressão do programa de treinamento também são discutíveis, sendo necessários estudos com desenhos melhor apropriados para a avaliação dessas variáveis. A seguir, citaremos a prescrição de exercícios nos idosos conforme alguns consensos encontrados na literatura:

\section{1) Avaliação pré-participação}

Contra-indicações estão presentes na tabela 1, mas no geral não diferem daquelas aplicadas ao adulto jovem $^{(60)}$. A necessidade de eletrocardiograma de esforço é contraditória; ela deve ser considerada para pacientes com fatores de risco cardiológicos.

\section{2) Como iniciar}

Os exercícios devem objetivar a melhora das limitações funcionais que o idoso venha a apresentar (dor, amplitude de movimento reduzida ou fraqueza muscular). Logo que houver melhora dessas limitações, um programa de condicionamento geral deve ser feito com o objetivo de implementar a saúde e a capacidade funcional do idoso.

As sessões de exercícios devem conter três fases: aquecimento, que envolve exercícios para o ganho de amplitude articular, sendo de baixa intensidade; período de treinamento (o esforço propriamente dito), que envolve o fortalecimento muscular e/ou exercícios aeróbicos, e a fase final que abrange basicamente alongamentos (desaquecimento).

\section{3) Alongamentos}

Os alongamentos devem ser realizados durante o aquecimento e na fase final. Grande amplitude de movimento (ADM) da articulação aumenta o desempenho muscular ${ }^{(61)}$, reduz o risco de lesão ${ }^{(61,62)}$ e melhora a nutrição da cartilagem ${ }^{(63)}$. Articulações dolorosas não devem ser alongadas de forma excessiva a ponto de causar dor; todos os movimentos devem ser feitos de modo a conseguir a máxima ADM livre de dor. A aplicação de calor antes do alongamento reduz a dor e aumenta a amplitude. Devem-se realizar pelo menos três sessões de alongamentos por semana ${ }^{(64)}$; no início, três a cinco repetições e aumentando gradualmente até 10 repetições ${ }^{(65)}$. Deve-se manter o músculo alongado durante 10 a 30 segundos.

\section{4) Fortalecimento muscular}

O fortalecimento muscular deve ser feito com uso de pesos ou faixas elásticas. que darão resistência ao movimento. Os protocolos de treinamento devem conter os seguintes princípios:

- exercícios de contração muscular devem ser feitos em moderada velocidade;

- os exercícios devem ser escolhidos de acordo com a estabilidade das articulações e o grau de dor e edema;

- os músculos não devem ser exercitados até sua fadiga;

- a resistência do exercício deve ser submáxima;

- articulações com reações inflamatórias devem ser fortalecidas com exercícios isométricos e envolvendo poucas repetições primeiramente;

- dor ou edema numa articulação após uma hora da realização dos exercícios indica atividade excessiva.

Exercícios isométricos são indicados para articulações instáveis ou edemaciadas. Contrações isométricas produzem baixa pressão articular e são bem toleradas por pa- 
cientes idosos $^{(66)}$. Inicia-se com contrações de intensidade aproximadamente de $30 \%$ da força máxima, aumentando gradativamente até $75 \%$. A contração não deve ser mantida por mais de seis a 10 segundos e as repetições devem ser aumentadas para de oito a 10, conforme tolerado pelo paciente. Deve ser realizada durante duas vezes por dia durante o período inflamatório e, após a inflamação retroceder, aumentar de cinco a 10 vezes por dia.

Os exercícios isotônicos devem ser de oito a 10 exercícios envolvendo os grupos musculares maiores (quatro exercícios para membros superiores e de -quatro a seis para membros inferiores). Devem-se usar inicialmente pesos com $40 \%$ da carga máxima do indivíduo, aumentando até para $80 \%{ }^{(67,68)}$. Geralmente, realiza-se uma série de quatro a seis repetições, evitando a fadiga muscular. A frequência deve ser de no máximo duas vezes por semana inicialmente ${ }^{(8)}$, podendo ser somente uma vez nos idosos com idade avançada ou com fragilidade importante. Deve haver pelo menos um dia de repouso inteiro entre as sessões ${ }^{(69)}$.

\section{5) Exercícios aeróbios}

O paciente pode escolher entre uma variedade de opções de exercícios, evitando sobrecarregar articulações especificas. Exemplos de exercícios são: andar de bicicleta, natação, exercícios de baixo impacto (caminhada, dança, tai chi chuan) e hidroterapia e até atividades mais leves como andar com o cachorro e/ou jogar golfe. A intensidade do exercício pode ser medida pela frequência cardíaca (FC) durante o exercício, devendo estar entre 50 e $75 \%$ da FC máxima (calculada como 220 menos os anos de idade); ou um talk test sendo considerado posi-

\section{REFERÊNCIAS}

1. Grimby G. Muscle performance and structure in the elderly as studied crosssectionally and longitudinally. J Gerontol A Biol Sci Med Sci. 1995;50(Spec):17-22.

2. Cress ME, Buchner DM, Prohaska T, Rimmer J, Brown M, Macera C, et al. Physical activity programs and behavior counseling in older adult populations. Med Sci Sports Exerc. 2004;36(11):1997-2003.

3. IBGE. Projeção da População do Brasil para o período 2000-2050. Disponível em: http://www.ibge.gov.br

4. Prevalence and impact of arthritis among women - United States, 1989- 1991. MMWR Morb Mortal Wkly Rep. 1995;44:331-335.

5. Frontera WR, Meredith CN, O'Reilly KP, Knuttgen HG, Evans WJ. Strength conditioning in older men: skeletal muscle hypertrophy and improved function. J Appl Physiol. 1988;64(3):1038-44.

6. Verbrugge LM. Women, men and osteoarthritis. Arth Care Res. 1995;8(4):212-20.

7. Felson DT, Naimark A, Anderson J, Kazis L, Castelli W, Meenan RF. The prevalence of knee osteoarthritis in the elderly: The Framingham Osteoarthritis Study. Arth Rheum. 1987;30(8):914-8.

8. American Geriatrics Society Panel on Exercise and Osteoarthritis. Exercise prescription for older adults with osteoarthritis pain: consensus practice recommendations. J Am Geriatr Soc. 2001;49(6):808-23.

9. Feder G, Cryer C, Donovan S, Carter Y. Guidelines for the prevention of falls in people over 65. The Guidelines' Dev Group. BMJ. 2000;321(7267):1007-11. tivo quando o paciente não consegue conversar confortavelmente durante a atividade física devido à alteração da frequência respiratória. É recomendado iniciar com duração de no mínimo 20 a 30 minutos por dia; durante a semana, o acumulado deve estar entre 60 e 90 minutos de atividades moderadas ${ }^{(8)}$. A frequência deve ser de pelo menos três e não mais do que quatro vezes por semana.

\section{6) Cuidados}

A pessoa com instabilidade postural ou com história de lesões por quedas deve ser encaminhada para fisioterapeutas, terapeutas ocupacionais ou educadores físicos qualificados para esse tipo de trabalho (reeducação postural e correção dos desequilíbrios) ${ }^{(69)}$. Tendinites, bursites e lombalgias podem ocorrer no idoso quando o fortalecimento é realizado de forma exagerada.

\section{CONCLUSÃO}

As alterações no aparelho locomotor ocorridas em decorrência do envelhecimento que causam perda no equilíbrio, fragilidade óssea, dores articulares e decréscimo da função podem ter seu efeito minimizado por meio da prática regular de exercícios físicos.

Apesar de haver muitas dúvidas quanto à melhor forma de prescrever e de orientar a atividade física, há tendência cada vez maior de associar exercícios aeróbios com exercícios resistidos (fortalecimento muscular). Os programas de exercícios devem ser individualizados conforme as necessidades específicas do idoso. A aderência a longo prazo ao programa proposto é necessária para que se mantenham os benefícios ganhos pela prática da atividade física.

10. Gregg EW, Pereira MA, Caspersen CJ. Physical activity, falls, and fractures among older adults: a review of the epidemiologic evidence. J Am Geriatr Soc. 2000;48(8):883-3.

11. Lord SR, Lloyd DG, Nirui M, Raymond J, Williams P, Stewart RA. The effect of exercise on gait patterns in older women: a randomized controlled trial. $\mathrm{J}$ Gerontol Biol Sci Med Sci. 1996;51(2):M64-70.

12. Skelton DA, Beyer N. Exercise and injury prevention in older people. Scand J Med Sci Sports. 2003;13(1):77-85.

13. Lindle RS, Metter EJ, Lynch NA, Fleg JL, Fozard JL, Tobin J, et al. Age and gender comparisons of muscle strength in 654 women and men aged 20-93 yr. J Appl Physiol. 1997; 83(5):1581-7.

14. Harridge S, Magnusson G, Saltin B. Life-long endurance-trained elderly men have high aerobic power, but have similar muscle strength to non-active elderly men. Aging (Milano). 1997;9(1-2):80-7.

15. Bonnefoy M, Cornu C, Normand S, Boutitie F, Bugnard F, Rahmani A, et al. The effects of exercise and protein-energy supplements on body composition and muscle function in frail elderly individuals: a longterm controlled randomised study. Br J Nutr. 2003;89(5):731-9.

16. Roubenoff R. Origins and clinical relevance of sarcopenia. Can J Appl Physiol. 2001;26(1):78-89.

17. Kamen G. Aging, resistance training, and motor unit discharge behaviour. Can J Appl Physiol. 2005;30(3):341-51. 
18. Skelton DA, Greig CA, Davies JM, Young A. Strength, power and related functional ability of healthy people aged 65-89 years. Age Ageing. 1994;23(5): 371-7.

19. Petrella JK, Kim JS, Tuggle SC, Hall SR, Bamman MM. Age differences in knee extension power, contractile velocity, and fatigability. J Appl Physiol. 2005;98(1):211-20

20. Fleck S, Kraemer JK. Designing resistance training programs. Champaign,IL: Human Kinetics; 2006.

21. Hurley MV. The role of muscle weakness in the pathogenesis of osteoarthritis. Rheum Dis Clin North Am. 1999;25(2):283-98.

22. Pai YC, Rymer WZ, Chang RW, Chang RW, Sharma L. Effect of age and osteoarthritis on knee proprioception. Arthritis Rheum. 1997;40(12):2260-5.

23. Hurley MV, Scott DL, Rees J, Newham DJ. Sensorimotor changes and functional performance in patients with knee osteoarthritis. Ann Rheum Dis. 1997;56(11):641-648.

24. Plapper PG. Avaliação numérica das ondulações das fibras colágenas em ligamento patelar humano [tese]. São Paulo: Instituto de Ortopedia e Traumatologia da Faculdade de Medicina, Universidade de São Paulo; 1997.

25. Consensus Development Conference. Diagnosis, prophylaxis and treatment of osteoporosis. American Journal of Medicine. 1993;94(6):646-50.

26. Ettinger WH Jr., Burns R, Messier SP, Applegate W, Rejeski WJ, Morgan T et al. A randomized trial comparing aerobic exercise with resistance exercise with a health education program in older adults with knee osteoarthritis: The Fitness Arthritis and Seniors Trial (FAST). JAMA. 1997;277(1):25-31.

27. Kovar PA, Allegrante JP, MacKenzie CR, Peterson MG, Gutin B, Charlson ME. Supervised fitness walking in patients with osteoarthritis of the knee. A randomized controlled trial. Ann Intern Med. 1992;116(7):529-34.

28. Lyngberg KK, Harreby M, Bentzen H, Frost B, Danneskiold-Samsoe B. Elderly rheumatoid arthritis patients on steroid treatment tolerate physical training without an increase in disease activity. Arch Phys Med Rehabil. 1994;75(11):1189-95.

29. Coleman EA, Buchner DM, Cress ME, Chan BK, de Lateur BJ. The relationship of joint symptoms with exercise performance in older adults. J Am Geriatr Soc. 1996;44(1):14-21.

30. Fransen M, McConnell S, Bell M. Exercise of osteoarthritis of the hip or knee. Cochrane Database Syst Rev. 2003;(3):CD004286.

31. Messier SP, Thompson CD, Ettinger Jr. WH. Effects of long-term aerobic or weight training regimens on gait in an older osteoarthritic population. $\mathrm{J} \mathrm{Appl}$ Biomech. 1997;13(2):205-25.

32. Messier SP, Royer TD, Craven TE, O'Toole ML, Burns R, Ettinger Wh Jr. Long-term exercise and its effect on balance in older, osteoarthritic adults: results from Fitness, Arthritis, and Seniors Trial (FAST). J Am Geriatr Soc. 2000;48(2):131-8.

33. Fransen M, McConnell S. Exercise for osteoarthritis of the knee. Cochrane Database Syst Rev. 2008 8;(4) ):CD004376.

34. Roddy E, Zhang W, Doherty M. Aerobic walking or strengthening exercise for osteoarthritis of the knee? A systematic review. Ann Rheum Dis. 2005;64(4):544-8.

35. Woodard CM, Berry MJ. Enhancing adherence to prescribed exercise: structured behavioral interventions in clinical exercise programs. J Cardiopulmonary Rehabilitation. 2001;21(4):201-9.

36. Hernández-Molina G, Reichenbach S, Zhang B, Lavalley M, Felson DT. Effect of therapeutic exercise for hip osteoarthritis pain: results of a meta-analysis. Arthritis Rheum. 2008 Sep 15;59(9):1221-8.

37. Tak E, Staats P, Van Hespen A, Hopman-Rock M. The effects of an exercise program for older adults with osteoarthritis of the hip. J Rheumatol. 2005 Jun;32(6):1106-13.

38. Bartels EM, Lund H, Hagen KB, Dagfinrud H, Christensen R, DanneskioldSamsøe B. Aquatic exercise for the treatment of knee and hip osteoarthritis. Cochrane Database Syst Rev. 2007;(4):CD005523.

39. Hagerman FC, Walsh SJ, Staron RS, Hikida RS, Gilders RM, Murray TF, et al. Effects of high-intensity resistance training on untrained older men. I. Strength, cardiovascular, and metabolic responses. J Gerontol A Biol Sci Med Sci. 2000;55(7):B336-46.

40. Harris C, DeBeliso MA, Spitzer-Gibson TA, Adams KJ. The effect of resistancetraining intensity on strength-gain response in the older adult. J Strength Cond Res. 2004;18(4):833-8

41. Krebs DE, Scarborough DM, McGibbon CA. Functional vs. strength training in disabled elderly outpatients. Am J Phys Med Rehabil. 2007; 86(2):93-103.

42. Narici MV, Reeves ND, Morse Cl, Maganaris CN. Muscular adaptations to resistance exercise in the elderly. J Musculoskelet Neuronal Interact. 2004;4(2):161-4.
43. Reeves ND, Narici MV, Maganaris CN. Musculoskeletal adaptations to resistance training in old age. Man Ther. 2006;11(3):192-6.

44. Miszko TA, Cress ME, Slade JM, Covey CJ, Agrawal SK, Doerr CE. Effect of strength and power training on physical function in community-dwelling older adults. J Gerontol A Biol Sci Med Sci. 2003; 58(2):171-5.

45. Porter MM. Power training for older adults. Appl Physiol Nutr Metab. 2006;31(2):87-94.

46. Skelton DA, Kennedy J, Rutherford OM. Explosive power and asymmetry in leg muscle function in frequent fallers and non-fallers aged over 65. Age Ageing. 2002; 31(2):119-25.

47. McMurdo ME, Rennie LM. Improvements in quadriceps strength with regular seated exercise in the institutionalized elderly. Arch Phys Med Rehabil. 1994; 75(5):600-3.

48. Jette AM, Harris BA, Sleeper L, Lachman ME, Heislein D, Giorgetti M, et al. A home-based exercise program for nondisabled older adults. J Am Geriatr Soc. 1996; 44(6):644-9.

49. Krebs DE, Jette AM, Assmann SF. Moderate exercise improves gait stability in disabled elders. Arch Phys Med Rehabil. 1998; 79(12):1489-95.

50. Orr R, Raymond J, Fiatarone Singh M. Efficacy of progressive resistance training on balance performance in older adults: a systematic review of randomized controlled trials. Sports Med. 2008;38(4):317-43.

51. Howe TE, Rochester L, Jackson A, Banks PM, Blair VA. Exercise for improving balance in older people. Cochrane Database Syst Rev. 2007 ;(4):CD004963.

52. Rutherford OM. Is there a role for exercise in the prevention of osteoporotic fractures? Br J Sports Med. 1999;33(6):378-86.

53. Kerr D, Morton A, Dick I, Prince R. Exercise effects on bone mass in postmenopausal women are site-specific and load-dependent. J Bone Miner Res. 1996; 11(2):218-25

54. Kohrt WM, Snead DB, Slatopolsky E, Birge SJ. Additive effects of weight-bearing exercise and estrogen on bone mineral density in older women. J Bone Miner Res. 1995;10(9):1303-11.

55. Welsh L, Rutherford OM. Hip bone mineral density is improved by high-impact aerobic exercise in postmenopausal women and men over 50 years. Eur $\mathrm{J}$ Appl Physiol Occup Physiol. 1996;74(6):511-7.

56. Nakatsuka K, Kawakami H, Miki T. Exercise and physical therapy in osteoporosis. Nippon Rinsho. 1994; 52(9):2360-6.

57. Bonaiuti D, Shea B, lovine R, Negrini S, Robinson V, Kemper HC, et al. Exercise for preventing and treating osteoporosis in postmenopausal women. Cochrane Database Syst Rev. 2002;(3):CD000333.

58. Korpelainen R, Keinänen-Kiukaanniemi S, Heikkinen J, Väänänen K, Korpelainen J. Effect of impact exercise on bone mineral density in elderly women with low BMD: a population-based randomized controlled 30-month intervention. Osteoporos Int. 2006;17(1):109-18.

59. Karinkanta S, Heinonen A, Sievänen H, Uusi-Rasi K, Fogelholm M, Kannus $P$. Maintenance of exercise-induced benefits in physical functioning and bone among elderly women. Osteoporos Int. 2009;20(4):665-74.

60. Nordemar R, Ekblom B, Zachrisson L, Lundqvist K. Physical training in rheumatoid arthritis: a controlled long-term study. Scand J Rheumatol.1981;10(1):17-23.

61. Noreau L, Moffett H, Drolet M, Parent E. Dance-based exercise program in rheumatoid arthritis. Am J Phys Med Rehabil.1997;76(2):109-13.

62. Oddis C. New perspectives on osteoarthritis. Am J Med.1996;100(2A): S10-15.

63. O'Reilly SC, Muir KR, Doherty M. Effectiveness of home exercise on pain and disability from osteoarthritis of the knee: a randomized controlled trial. Ann Rheum Dis.1999;58(1):15-9.

64. Pollock ML, Mengelkoch LJ, Graves JE, Lowenthal DT, Limacher MC, Foster C, et al. Twenty-year follow-up of aerobic power and body composition of older track athletes. J Appl Physiol. 1997;82(5):1508-16.

65. Lindh M. Increase of muscle strength from isometric quadriceps exercise at different knee angles. Scand J Rehab Med.1979;11(1):33-6.

66. Rogind H, Bibow-Nielsen B, Jensen B, Moller HC, Frimodt-Moller H, Bliddal H. The effects of a physical training program on patients with osteoarthritis of the knees. Arch Phys Med Rehabil. 1998;79(11):1421-7.

67. de Vos NJ, Singh NA, Ross DA, Stavrinos TM, Orr R, Fiatarone Singh MA. Optimal load for increasing muscle power during explosive resistance training in older adults. J Gerontol A Biol Sci Med Sci. 2005;60(5):638-47.

68. Vincent KR, Braith RW, Feldman RA, Magyari PM, Cutler RB, Persin SA, et al. Resistance exercise and physical performance in adults aged 60 to 83. J Am Geriatr Soc. 2002;50(6):1100-7.

69. Hautier C, Bonnefoy M. Training for older adults. Ann Readapt Med Phys. 2007;50(6):475-9. 